\title{
NOTE
}

\section{Multiple trophic levels in soft-bottom communities}

\author{
John A. Commito ${ }^{1.2} \&$ William G. Ambrose, Jr. ${ }^{2}$ \\ ${ }^{1}$ Department of Biology, Hood College, Frederick, Maryland 21701, USA \\ ${ }^{2}$ Department of Marine Zoology and Marine Chemistry, University of Oslo, Oslo 3, Norway
}

\begin{abstract}
In order to assess the general applicability of recent field experiments with predatory infauna, we searched the literature and found 48 well-documented cases of infaunal consumption by such predators. In $63 \%$ of the cases detailed enough to make a determination, the predators ate other predators. Multiple trophic levels within the infauna are probably a common feature of many soft-bottom communities.
\end{abstract}

The roles which predatory infauna play in controlling soft-bottom community structure are poorly understood (see Commito \& Ambrose [in press] for review). Recent field experiments have demonstrated that predatory infauna (organisms which live within the sediment and ingest benthic animals) are important regulating agents and that multiple trophic levels exist within the infauna (Reise 1979, Commito 1982a, Commito \& Shrader in press, Ambrose 1984a,b,c). In order to assess the general applicability of these field experiment results, we searched the literature for evidence of infaunal consumption by predatory infauna. Only results from gut and fecal analyses, feeding trials, laboratory and field observations, and immunological assays were included. Examples of predation inferred from field manipulations were excluded because predatory infauna may have effects other than direct predation mortality. Such effects include induced emigration of prey, sublethal effects on prey, nutrient enrichment from fecal material, sediment modification via creation of biogenic structures, and sediment disturbances (Kneib \& Stiven 1982, Commito \& Ambrose in press, Commito \& Shrader in press).

We found 48 well-documented cases of predation by infaunal polychaetes, nemerteans, amphipods, tanaids, and gastropods on other benthic organisms (Table 1). Polynoid polychaetes and naticid and melongenid gastropods were considered predatory infauna even though they spend considerable time on the sediment surface. Our findings suggest that predatory infauna are important in many marine communities. Based primarily on information provided in the works cited, we also included in the table an estimate of the sediment disturbance caused by each predator. Too little information was available to evaluate the other types of non-predation effects.

In 21 of the 48 cases it was impossible to determine whether or not the predators ate other predators; either prey categories were too general (e.g. 'polychaetes') or researchers did not offer predatory infauna as prey to their test animals in feeding trials. In 17 (63\%) of the remaining 27 cases, the predators consumed other predators. We feel that many predatory infaunal species eat other predators and that the existence of multiple trophic levels within the infauna may be a common phenomenon in soft-bottom communities. If so, then analyses of energy flow (Ankar 1977. Mann 1982, Fasham 1984) and community organization (Peterson 1979, Woodin 1983) must continue to be re-evaluated for marine ecosystems.

Acknowledgements. Both authors wish to thank the University of Oslo Department of Marine Zoology and Marine Chemistry for support during the preparation of this paper Additional support came from a BP Petroleum Development Ltd grant to the University of Oslo, a Hood College Board of Associates Faculty Development grant to J. A. C., and a Royal Norwegian Council for Scientific and Industrial Research postdoctoral fellowship to W. G. A., Jr.

\section{LITERATURE CITED}

Ambrose, W. G., Jr. (1984a). Role of predatory infauna in structuring marine soft-bottom communities. Mar. Ecol. Prog. Ser. 17: 109-115

Ambrose, W. G., Jr. (1984b). Influences of predatory polychaetes and epibenthic predators on the structure of a soft-bottom community in a Maine estuary. J. exp. mar Biol. Ecol. 81: 115-145

Ambrose, W. G., Jr. (1984c). Influence of residents on the development of a marine soft-bottom community. J. mar. Res. 42: 633-654

Ambrose, W. G., Jr. (1984d). Increased emigration of the amphipod Rhepaxynius abronius (Barnard) and the polychaete Nephtys caeca (Fabricius) in the presence of invertebrate predators. J. exp. mar. Biol. Ecol. 80: 67-75 
Table 1 Direct evidence of infaunal consumption by predatory infauna. Results from gut and fecal analyses, immunological assay, feeding trial, laboratory observation, and field observation studies

\begin{tabular}{|c|c|c|c|c|c|c|c|}
\hline Predator & $\begin{array}{l}\text { Life posi- } \\
\text { tion in } \\
\text { sediment }\end{array}$ & $\begin{array}{l}\text { Location } \\
\text { of prey } \\
\text { capture }\end{array}$ & $\begin{array}{l}\text { Sediment } \\
\text { disturb- } \\
\text { ance }\end{array}$ & Macrofaunal prey & $\begin{array}{l}\text { Method of prey } \\
\text { determination }\end{array}$ & Source & Comments \\
\hline \multicolumn{8}{|l|}{ POLYCHAETA } \\
\hline $\begin{array}{l}\text { Polynoidae } \\
\text { Harmothoe sarsi }\end{array}$ & $\begin{array}{l}\text { Surface, } \\
\text { shallow }\end{array}$ & $\begin{array}{l}\text { Surface, } \\
\text { shallow }\end{array}$ & Slight & $\begin{array}{l}\text { Amphipods (esp. } \\
\text { Pontoporeia affinis), } \\
\text { Macoma balthica, } \\
\text { Harmothoe sarsi, } \\
\text { oligochaetes }\end{array}$ & Gut analysis & Sarvala 1971 & $\begin{array}{l}\text { Diet changes with } \\
\text { age; large worms } \\
\text { feed slmost exclu- } \\
\text { sively on Pontoporela } \\
\text { affinis }\end{array}$ \\
\hline $\begin{array}{l}\text { Hesionidae } \\
\text { Nereimyra } \\
\text { punctata }\end{array}$ & Shallow & $\begin{array}{l}\text { Surface, } \\
\text { shallow }\end{array}$ & Slight & $\begin{array}{l}\text { Polychaetes (esp. } \\
\text { Prionospio spp.). } \\
\text { amphipods }\end{array}$ & $\begin{array}{l}\text { Gut and fecal } \\
\text { analyses, feed- } \\
\text { ing trials }\end{array}$ & Oug 1980 & $\begin{array}{l}\text { Lives in gallery; re- } \\
\text { sponds to tactile and } \\
\text { chemical cues from } \\
\text { prey }\end{array}$ \\
\hline $\begin{array}{l}\text { Ophiodromus } \\
\text { flexuosus }\end{array}$ & $\begin{array}{l}\text { Surface, } \\
\text { shallow }\end{array}$ & $\begin{array}{l}\text { Surface, } \\
\text { shallow }\end{array}$ & Moderate & $\begin{array}{l}\text { Polychaetes (esp. } \\
\text { Prionospiospp.), } \\
\text { crustaceans, gas- } \\
\text { tropods, bivalves }\end{array}$ & $\begin{array}{l}\text { Gut and fecal } \\
\text { analyses, feed- } \\
\text { ing trials }\end{array}$ & Oug 1980 & $\begin{array}{l}\text { Responds to moving } \\
\text { prey, creeps along } \\
\text { surface }\end{array}$ \\
\hline $\begin{array}{l}\text { Podarke } \\
\text { pugettensis }\end{array}$ & $\begin{array}{l}\text { Surface, } \\
\text { shallow }\end{array}$ & $\begin{array}{l}\text { Surface, } \\
\text { shallow }\end{array}$ & Moderate & $\begin{array}{l}\text { Polychaetes (11 } \\
\text { families), tanaids, } \\
\text { isopods }\end{array}$ & $\begin{array}{l}\text { Gut and fecal } \\
\text { andlyses, feed- } \\
\text { ing trials }\end{array}$ & Shaffer 1979 & $\begin{array}{l}\text { Responds to moving } \\
\text { prey; usually rejects } \\
\text { large prey }\end{array}$ \\
\hline \multicolumn{8}{|l|}{ Nereidae } \\
\hline $\begin{array}{l}\text { Nereis } \\
\text { diversicolor }\end{array}$ & $\begin{array}{l}\text { Surface, } \\
\text { shallow, } \\
\text { deep }\end{array}$ & $\begin{array}{l}\text { Shallow, } \\
\text { deep }\end{array}$ & Slight & Arenicola marina & $\begin{array}{l}\text { Laboratory ob- } \\
\text { servation }\end{array}$ & $\begin{array}{l}\text { Witte \& de } \\
\text { Wilde } 1979\end{array}$ & $\begin{array}{l}\text { Invades prey bur- } \\
\text { rows; bites off tails or } \\
\text { kills prey }\end{array}$ \\
\hline $\begin{array}{l}\text { Nereis } \\
\text { diversicolor }\end{array}$ & Shallow & Surface & Moderate & Corophium volutator & $\begin{array}{l}\text { Laboratory ob- } \\
\text { servation }\end{array}$ & $\begin{array}{l}\text { Olafsson \& } \\
\text { Persson un- } \\
\text { publ. }\end{array}$ & $\begin{array}{l}\text { Disturbs prey bur- } \\
\text { rows; attacks prey } \\
\text { only on sediment sur- } \\
\text { face }\end{array}$ \\
\hline Nereis succinea & $\begin{array}{l}\text { Surface, } \\
\text { shallow. } \\
\text { deep }\end{array}$ & $\begin{array}{l}\text { Surface, } \\
\text { shallow }\end{array}$ & Moderate & $\begin{array}{l}\text { Amphipods (esp. } \\
\text { Corophium lacustre, } \\
\text { Leptocheirus } \\
\text { plumulosus), nereid } \\
\text { polychaetes }\end{array}$ & Gut analysis & $\begin{array}{l}\text { Haddon \& } \\
\text { Hines unpubl. }\end{array}$ & $\begin{array}{l}\text { Feeds primarily on } \\
\text { surface organisms } \\
>1 \mathrm{~mm} \text { long }\end{array}$ \\
\hline Nereis virens & $\begin{array}{l}\text { Surface, } \\
\text { shallow, } \\
\text { deep }\end{array}$ & $\begin{array}{l}\text { Surface, } \\
\text { shallow }\end{array}$ & Moderate & $\begin{array}{l}\text { Polychaetes, } \\
\text { oligochaetes, } \\
\text { bivalves, amphipods }\end{array}$ & Fecal analysis & Ambrose 1984b & $\begin{array}{l}\text { Moves anterior part } \\
\text { of body out of burrow } \\
\text { across sediment sur- } \\
\text { face to attack prey }\end{array}$ \\
\hline \multicolumn{8}{|l|}{ Nephtyidae } \\
\hline Nephtys caeca & $\begin{array}{l}\text { Shallow, } \\
\text { deep }\end{array}$ & $\begin{array}{l}\text { Shallow, } \\
\text { deep }\end{array}$ & Slight & $\begin{array}{l}\text { Rhepoxynius ab- } \\
\text { ronius }\end{array}$ & Feeding trials & Ambrose $1984 d$ & $\begin{array}{l}\text { Causes prey to leave } \\
\text { sediment and enter } \\
\text { water column }\end{array}$ \\
\hline Nephtys cirrosa & $\begin{array}{l}\text { Shallow, } \\
\text { deep }\end{array}$ & $\begin{array}{l}\text { Shallow, } \\
\text { deep }\end{array}$ & Slight & $\begin{array}{l}\text { Polychaetes (Neph- } \\
\text { tys spp., possibly } \\
\text { maldanids and } \\
\text { spionids) }\end{array}$ & Gut analysis & Clark 1962 & $\begin{array}{l}\text { Many guts empty or } \\
\text { containing sediment }\end{array}$ \\
\hline Nephtys hombergi & $\begin{array}{l}\text { Shallow, } \\
\text { deep }\end{array}$ & $\begin{array}{l}\text { Shallow, } \\
\text { deep }\end{array}$ & Slight & $\begin{array}{l}\text { Polychaetes (esp. } \\
\text { spionids) }\end{array}$ & Gut analysis & Clark 1962 & $\begin{array}{l}\text { Many guts empty or } \\
\text { containing sediment }\end{array}$ \\
\hline \multicolumn{8}{|l|}{ Glyceridae } \\
\hline Glyceraalba & $\begin{array}{l}\text { Shallow, } \\
\text { deep }\end{array}$ & $\begin{array}{l}\text { Surface, } \\
\text { shallow }\end{array}$ & Slight & $\begin{array}{l}\text { Many polychaete } \\
\text { species (esp. errant } \\
\text { forms), amphipods }\end{array}$ & $\begin{array}{l}\text { Fecal analysis, } \\
\text { feeding trals }\end{array}$ & $\begin{array}{l}\text { Ockelmann \& } \\
\text { Vahl } 1970\end{array}$ & $\begin{array}{l}\text { Responds to moving } \\
\text { prey; lives in gallery; } \\
\text { injects toxin }\end{array}$ \\
\hline Glycera convoluta & $\begin{array}{l}\text { Shallow, } \\
\text { deep }\end{array}$ & $\begin{array}{l}\text { Surface, } \\
\text { shaitow }\end{array}$ & Slight & Nephtys caeca & Feeding trials & Ambrose $1984 d$ & $\begin{array}{l}\text { Lives in gallery; } \\
\text { causes prey to leave } \\
\text { sediment and enter } \\
\text { water column }\end{array}$ \\
\hline $\begin{array}{l}\text { Glycera } \\
\text { dibranchiata }\end{array}$ & $\begin{array}{l}\text { Shallow, } \\
\text { deep }\end{array}$ & $\begin{array}{l}\text { Surface, } \\
\text { shallow }\end{array}$ & Slight & $\begin{array}{l}\text { Nereis virens, } \\
\text { Macoma balthica, } \\
\text { Corophium volutator }\end{array}$ & $\begin{array}{l}\text { Fecal analysis, } \\
\text { feeding trials }\end{array}$ & Ambrose $1984 b$ & $\begin{array}{l}\text { Lives in gallery; in- } \\
\text { jects toxin }\end{array}$ \\
\hline $\begin{array}{l}\text { Splonidae } \\
\text { Pseudopolydora } \\
\text { kempi }\end{array}$ & Shallow & Surface & Moderate & Abarenicola pacifica & $\begin{array}{l}\text { Gutanalysis, } \\
\text { feeding trials }\end{array}$ & Wilson 1981 & $\begin{array}{l}\text { Feeds on juvenile } \\
\text { worms }\end{array}$ \\
\hline Pygospro elegans & Shallow & Surface & Moderate & Abarenicola pacifica & $\begin{array}{l}\text { Gut analysis, } \\
\text { feeding trials }\end{array}$ & Wilson 1981 & $\begin{array}{l}\text { Feeds on juvenile } \\
\text { worms }\end{array}$ \\
\hline $\begin{array}{l}\text { Terebellidae } \\
\text { Eupolymmia } \\
\text { heterobranchia }\end{array}$ & $\begin{array}{l}\text { Shallow, } \\
\text { deep }\end{array}$ & Surface & Moderate & $\begin{array}{l}\text { Polychaetes, } \\
\text { amphipods }\end{array}$ & $\begin{array}{l}\text { Fecal analysis, } \\
\text { feeding trials, } \\
\text { laboratory ob- } \\
\text { servation }\end{array}$ & Wilson 1980 & Feeds on small forms \\
\hline
\end{tabular}


Table 1. (Continued)

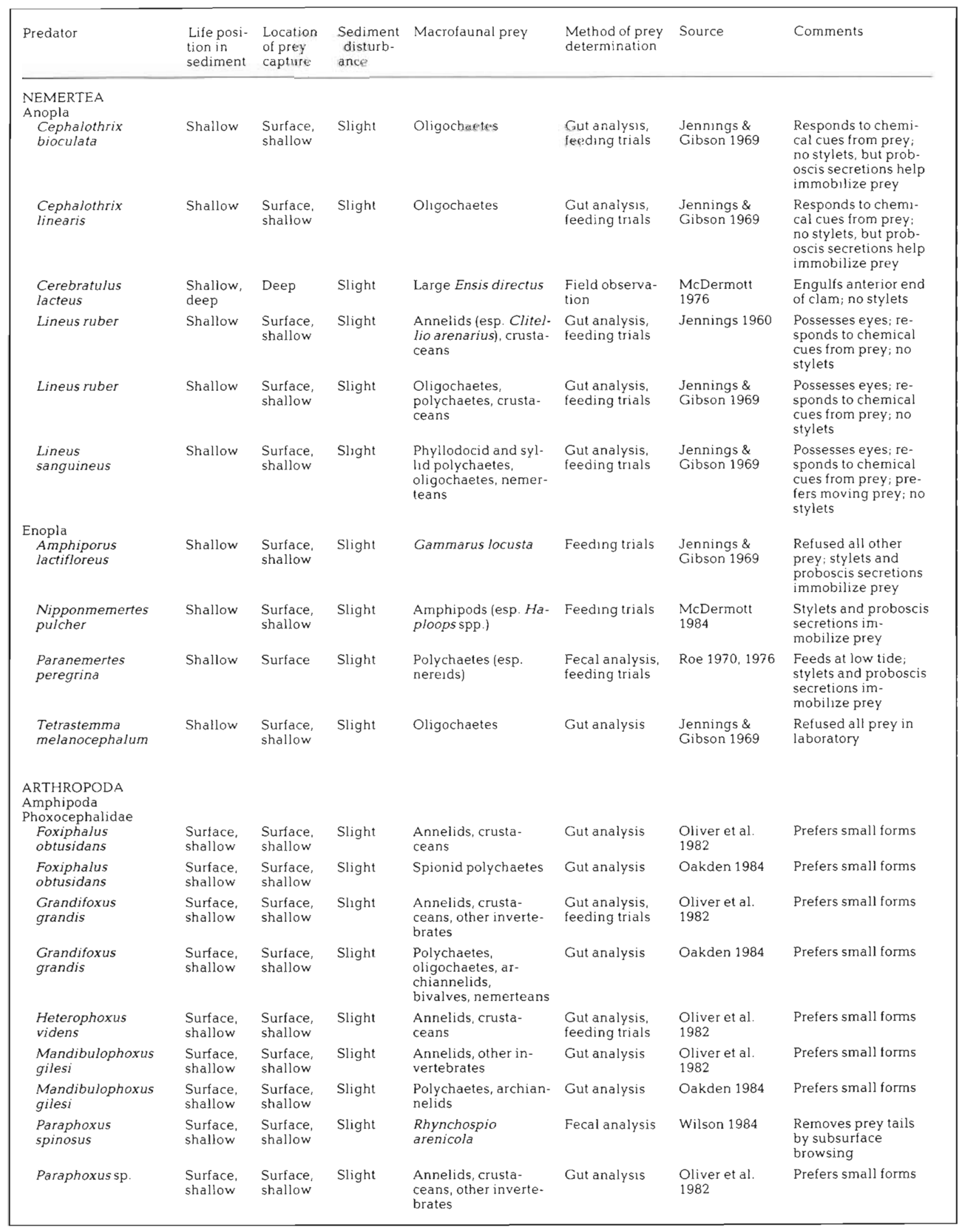


Table 1. (Continued)

\begin{tabular}{|c|c|c|c|c|c|c|c|}
\hline Predator & $\begin{array}{l}\text { Life posi- } \\
\text { tion in } \\
\text { sediment }\end{array}$ & $\begin{array}{l}\text { Location } \\
\text { of prey } \\
\text { capture }\end{array}$ & $\begin{array}{l}\text { Sediment } \\
\text { disturb- } \\
\text { ance }\end{array}$ & Macrofaunal prey & $\begin{array}{l}\text { Method of prey } \\
\text { determination }\end{array}$ & Source & Comments \\
\hline $\begin{array}{l}\text { Rhepoxynus } \\
\text { abronius }\end{array}$ & $\begin{array}{l}\text { Surface, } \\
\text { shaîiow }\end{array}$ & $\begin{array}{l}\text { Surface, } \\
\text { shallow }\end{array}$ & Slight & $\begin{array}{l}\text { Polychaetes, gas- } \\
\text { tropods }\end{array}$ & Gut analysis & Oakden 1984 & Prefers sma!l forms \\
\hline $\begin{array}{l}\text { Rhepoxynius } \\
\text { daboius }\end{array}$ & $\begin{array}{l}\text { Surface, } \\
\text { shallow }\end{array}$ & $\begin{array}{l}\text { Surface, } \\
\text { shallow }\end{array}$ & Slight & $\begin{array}{l}\text { Annelids, crusta- } \\
\text { ceans, other inverte- } \\
\text { brates }\end{array}$ & Gut analysis & $\begin{array}{l}\text { Oliver el al. } \\
1982\end{array}$ & Prefers small forms \\
\hline $\begin{array}{l}\text { Rhepoxynius } \\
\text { epistomus }\end{array}$ & $\begin{array}{l}\text { Surface, } \\
\text { shallow }\end{array}$ & $\begin{array}{l}\text { Surface, } \\
\text { shallow }\end{array}$ & Slight & $\begin{array}{l}\text { Annelids, other in- } \\
\text { vertebrates }\end{array}$ & $\begin{array}{l}\text { Gut analysis } \\
\text { feeding trials }\end{array}$ & $\begin{array}{l}\text { Oliver etal. } \\
1982\end{array}$ & Prefers small forms \\
\hline $\begin{array}{l}\text { Rhepoxynius } \\
\text { fatigans }\end{array}$ & $\begin{array}{l}\text { Surface, } \\
\text { shallow }\end{array}$ & $\begin{array}{l}\text { Surface, } \\
\text { shallow }\end{array}$ & Slight & $\begin{array}{l}\text { Polychaetes, crusta- } \\
\text { ceans }\end{array}$ & Gut analysis & Oakden 1984 & Prefers small forms \\
\hline $\begin{array}{l}\text { Corophildae } \\
\text { Corophium } \\
\text { salmons }\end{array}$ & $\begin{array}{l}\text { Surface, } \\
\text { shallow }\end{array}$ & $\begin{array}{l}\text { Surface, } \\
\text { shallow }\end{array}$ & Moderate & $\begin{array}{l}\text { Polychaetes, } \\
\text { bivalves, crusta- } \\
\text { coans, nemerteans }\end{array}$ & $\begin{array}{l}\text { Immunoassay } \\
\text { of gut contents }\end{array}$ & $\begin{array}{l}\text { Feller et al. } \\
1979\end{array}$ & $\begin{array}{l}\text { Accuracy of im- } \\
\text { munoassay techni- } \\
\text { que not yet fully es- } \\
\text { tablished }\end{array}$ \\
\hline $\begin{array}{l}\text { Gammaridae } \\
\text { Pontoporeia } \\
\text { affinis }\end{array}$ & $\begin{array}{l}\text { Surface, } \\
\text { shallow }\end{array}$ & $\begin{array}{l}\text { Surface, } \\
\text { shallow }\end{array}$ & Moderate & Mytilus edulis & Foeding trials & $\begin{array}{l}\text { Segestråle } \\
1962\end{array}$ & $\begin{array}{l}\text { Ingests bivalve lar- } \\
\text { vae, probably Maco- } \\
\text { ma balthica in field }\end{array}$ \\
\hline $\begin{array}{l}\text { Tanaidacea } \\
\text { Leptochelia dubia }\end{array}$ & $\begin{array}{l}\text { Surface, } \\
\text { shallow }\end{array}$ & Surface & Moderate & $\begin{array}{l}\text { Dendraster } \\
\text { excentricus }\end{array}$ & Foeding trials & $\begin{array}{l}\text { Highsmith } \\
1982\end{array}$ & $\begin{array}{l}\text { Females capture lar- } \\
\text { yae and juvenile } \\
\text { forms }\end{array}$ \\
\hline $\begin{array}{l}\text { MOLLUSCA } \\
\text { Gastropoda } \\
\text { Naticidae }\end{array}$ & & & & & & & \\
\hline Lunatia heros & $\begin{array}{l}\text { Shallow, } \\
\text { deep }\end{array}$ & $\begin{array}{l}\text { Shallow, } \\
\text { deep }\end{array}$ & Slight & $\begin{array}{l}\text { Mya arenaria, } \\
\text { Macoma balthica }\end{array}$ & $\begin{array}{l}\text { Field observa- } \\
\text { tion }\end{array}$ & $\begin{array}{l}\text { Commito } \\
1982 b\end{array}$ & $\begin{array}{l}\text { Usually forages deep } \\
\text { below sediment sur- } \\
\text { face; drills bivalve } \\
\text { shells }\end{array}$ \\
\hline $\begin{array}{l}\text { Polinices } \\
\text { duplicatus }\end{array}$ & $\begin{array}{l}\text { Shallow, } \\
\text { deep }\end{array}$ & $\begin{array}{l}\text { Shallow, } \\
\text { deep }\end{array}$ & Severe & Mya arenaria & $\begin{array}{l}\text { Field observa- } \\
\text { tion }\end{array}$ & $\begin{array}{l}\text { Edwards \& } \\
\text { Huebner } 1977\end{array}$ & $\begin{array}{l}\text { Usually forages near } \\
\text { sediment surface; } \\
\text { leaves distinct trails; } \\
\text { drils bivalve shells }\end{array}$ \\
\hline $\begin{array}{l}\text { Melongenidae } \\
\text { Busyconspp. }\end{array}$ & $\begin{array}{l}\text { Surface, } \\
\text { shallow, } \\
\text { deep }\end{array}$ & $\begin{array}{l}\text { Shallow, } \\
\text { deep }\end{array}$ & Severe & $\begin{array}{l}\text { Mercenariamer- } \\
\text { cenarla, Chione can- } \\
\text { cellata }\end{array}$ & $\begin{array}{l}\text { Field obverva- } \\
\text { tion }\end{array}$ & Peterson 1982 & $\begin{array}{l}3 \text { ssp. } \\
\text { (canaliculatum, cari- } \\
\text { ca, contrarium) } \\
\text { analyzed as a group; } \\
\text { plow through sedi- } \\
\text { ment; break bivalve } \\
\text { shell margins }\end{array}$ \\
\hline
\end{tabular}

Ankar, S. (1977). The soft-bottom ecosystem of the Northern Baltic proper with special reference to the macrofauna. Contr from the Askø Lab., Univ. of Stockholm, Sweden 19: $1-62$

Clark, R. B. (1962). Observations on the food of Nephtys. Limnol. Oceanogr $7: 380-385$

Commito, J. A. (1982a). Importance of predation by infaunal polychaetes in controlling the structure of a soft-bottom community in Maine, USA. Mar Biol. 68: 77-81

Commito, J. A. (1982b). Effects of Lunatia heros predation on the population dynamics of Mya arenaria and Macoma balthica in Maine, USA. Mar Biol. 69: 187-193

Commito, J. A., Ambrose, W G., Jr. (in press). Predatory infauna and trophic complexity in soft-bottom communities. Proc of the 19th Eur. Mar. Biol. Symp., Cambridge University Press, Cambridge

Commito, J. A., Shrader, P. B (in press). Benthic community response to experimental additions of the polychaete Nereis virens. Mar Biol

Edwards, D. C., Huebner, J. D. (1977). Feeding and growth rates of Polinices duplicatus preying on Mya arenaria at Barnstable Harbor, Massachusetts. Ecology 58: 1218-1236

Fasham, M. J. R. (ed.) (1984). Flows of energy and materials in marine ecosystems. Plenum, New York
Feller, R. J., Taghon, G. L., Gallagher, E. D., Kenny, G. E., Jumars, P. A. (1979). Immunological methods for food web analysis in a soft-bottom benthic community. Mar Biol. 54: $61-74$

Highsmith, R. C. (1982). Induced settlement and metamorphosis of sand dollar (Dendraster excentricus) larvae in predator-free sites: adult sand dollar beds. Ecology 63: 329-337

Jennings, J. B. (1960). Observations on the nutrition of the rhyncocoelan Lineus ruber (O. F. Müller). Biol. Bull. mar biol. Lab., Woods Hole 119: 189-196

Jennings, J. B., Gibson, R. (1969). Observations on the nutrition of seven species of rhyncocoelan worms. Biol. Bull. mar biol. Lab., Woods Hole 136: 405-433

Kneib, R. J., Stiven, A. E. (1982). Benthic invertebrate response to size and density manipulations of the common mummichog, Fundulus heterocitus, in an intertidal salt marsh. Ecology 63: 1518-1532

Mann, K. H. (1982). Ecology of coastal waters. Blackwell Scientific Publications, Oxford

McDermott, J. J. (1976). Predation of the razor clam Ensis directus by the nemertean worm Cerebratulus lacteus. Chesapeake Sci. 17: 299-301

McDermott, J. J. (1984). The feeding biology of Nip- 
ponemertes pulcher (Johnston) (Haplonemertea) with some ecological implications. Ophelia 23: 1-21

Oakden, J. M. (1984). Feeding and substrate preference in five species of phoxocephalid amphipods from central California. J. Crust. Biol. 42: 233-247

Ockelmann, K. W., Vahl, O. (1970). On the bjology of the polychaete Glycera alba, especially its burrowing and feeding. Ophelia 8: 275-294

Oliver, J. S., Oakden, J. M., Slattery, P. N. (1982). Phoxocephalid amphipod crustaceans as predators on larvae and juveniles in marine soft-bottom communities. Mar. Ecol. Prog. Ser 7: 179-184

Oug, E. (1980). On feeding and behavior of Ophiodromus flexuosus (delle Chiaje) and Nereimyra punctata (O. F. Müller) (Polychaeta, Hesionidae). Ophelia 19: 175-192

Peterson, C. H. (1979). Predation, competitive exclusion, and diversity in the soft-sediment communities of estuaries and lagoons. In: Livingston, R. J. (ed.) Ecological processes in coastal and marine systems. Plenum, New York, p. 233-264

Peterson, C. H. (1982). Clam predation by whelks (Busycon spp.): experimental tests of the importance of prey size, prey density, and seagrass cover Mar Biol. 66: 159-170

Reise, K. (1979). Moderate predation on meiofauna by the macrobenthos of the Wadden Sea. Helgoländer Meeresunters. 32: 453-465

Roe, P. (1970). The nutrition of Paranemertes peregrina (Rhyncocolea: Hoplonemertea). I. Studies on food and feeding behavior Biol. Bull. mar. biol. Lab., Woods Hole 139: $80-91$

Roe, P. (1976). Life history and predator-prey interactions of the nemertean Paranemertes peregrina Coe. Biol. Bull. mar, biol. Lab., Woods Hole 150: 80-106

Sarvala, J. (1971). Ecology of Harmothoe sarsi (Malmgren) (Polychaeta, Polynoidae) in the northern Baltic area. Annls Zool fenn. 8: 231-309

Segerstråle, S. G., 1962. Investigations on Baltic populations of the bivalve Macoma baltica (L.). Part II. What are the reasons for the periodic failure of recruitment and the scarcity of Macoma in the deeper waters of the inner Baltic? Commentat. Biol. Soc Sci. Fenn. 24: 1-26

Shaffer, P. L. (1979). The feeding biology of Podarke pugettensis (Polychaeta: Hesionidae). Biol. Bull. mar. biol. Lab., Woods hole 156: 343-355

Wilson, W H., Jr. (1980). A laboratory investigation of the effect of a terebellid polychaete on the survivorship of nereid polychaete larvae. J. exp. mar. Biol. Ecol. 46: 73-80

Wilson, W. H. Jr. (1981). Sediment-mediated interactions in a densely populated infaunal assemblage: The effects of the polychaete Abarenicola pacifica. J. mar. Res. 39: 735-748

Wilson, W. H., Jr. (1984). An experimental analysis of spatial competition in a dense infaunal community: The importance of relative effects. Estuar. coast. Shelf Sci. 18: 673-684

Witte, F., Wilde, P. A. W. J. de (1979). On the ecological relation between Nereis diversicolor and juvenile Arenicola marina. Neth. J. Sea Res. 13: 394-405

Woodin, S. A. (1983). Biotic interactions in recent marine sedimentary environments. In: Tevesz, M. J. S., McCall, P. L. (ed.) Biotic interactions in recent and fossil benthic communities. Academic Press, New York, p. 3-38

Accepted for printing on June 18, 1985 